\title{
Bounded polyharmonic functions and the dimension of the manifold
}

\author{
By \\ Norman Mirsky, Leo SARIO, and Cecilia WANG
}

(Communicated by Professor Kusunoki, November 6, 1972)

Let $H^{2} B$ be the class of bounded biharmonic nonharmonic functions, i.e., nondegenerate solutions of $\Delta^{2} u=0$, with $\Delta$ the Laplace-Beltrami operator $d \delta+\delta d$. Consider the punctured space $E_{\alpha}^{N}: 0<|x|<\infty$, $x=\left(x^{1}, \ldots, x^{N}\right)$ with the metric $d s=|x|^{\alpha}|d x|, \alpha$ a constant. It was shown in Sario-Wang [1] that although $E_{\alpha}^{N}$ with $N=2,3$ carries $H^{2} B$-functions for infinitely many values of $a$, it tolerates no $H^{2} B$-functions for any $\alpha$ if $N \geq 4$. In the present paper we ask: What can be said about the class $H^{k} B$ of bounded nondegenerate polyharmonic functions of degree $k$, that is, solutions of $\Delta^{k} u=0$ ? The answer turns out to be rewarding and puts the biharmonic case in proper perspective: There exist no $H^{k} B$-functions on $E_{\alpha}^{N}$ for any a if $N \geq 2 k$.

For $N<2 k$ there are infinitely many a for which these functions do exist, and for these $a$ the generators of the space $H^{k} B$ are surface spherical harmonics. In particular, this is true of $H^{2} B$-functions on Euclidean 2- and 3-spaces, as was recently shown in Sario-Wang [2]. If $H^{k} B \neq \varnothing$ on a given $E_{\alpha}^{N}$, is the same true of $H^{h} B$ for any $h>k$ ? We shall show that, while this is so for every $N$ if the metric of $E_{\alpha}^{N}$ is Euclidean, there are values of $(N, \alpha)$ for which it does not hold.

AMS 1970 subject classification 31B30.

The work was sponsored by the U.S. Army Research Office-Durham, Grant DAARO-D-31-124-71-G181, University of California, Los Angeles. 
1. We start by stating our main result. Let $O_{H^{k}}^{N_{B}}$ be the class of Riemannian $N$-manifolds which do not carry bounded functions $u$ satisfying $\Delta^{k} u \equiv 0, \Delta^{h} u \neq 0$ for all $h<k$.

Theorem 1. $E_{\alpha}^{N} \in O_{H^{k}}^{N_{B}}$ for all $N \geq 2 k, k \geq 1$, and all $a$.

The proof will be given in Nos. 2-9

2. First we consider radial functions, i.e., those depending on $r=|x|$ only. We shall show that the equation $\Delta^{k} \gamma(r)=0$ has the following general solutions. If $N$ is odd, or if $N$ is even with $N>2 k$, then for any $\alpha \neq-1$.

$$
\gamma_{k}(r)=\sum_{n=0}^{k-1}\left(a_{n} r^{(2 n-N+2)(\alpha+1)}+b_{n} r^{2 n(\alpha+1)}\right)
$$

If $N$ is even with $N \leq 2 k$, then for any $a \neq-1$

$$
\gamma_{k}(r)=\sum_{n=0}^{k-1}\left(a_{n} r^{(2 n-N+2)(\alpha+1)}+b_{n} r^{2 n(\alpha+1)}\right)+\sum_{n=0}^{\frac{1}{2}(2 k-N)} c_{n} r^{2 n(\alpha+1)} \log r .
$$

If $a=-1$, then for any $N$

$$
\gamma_{k}(r)=\sum_{n=0}^{2 k-1} a_{n}(\log r)^{n}
$$

Since the proofs are similar in all cases, we shall only discuss the case $N$ odd, $a \neq-1$. For $f \in C^{2}\left(E_{\alpha}^{N}\right)$,

$$
\Delta f(r)=-\frac{1}{r^{N-1+N a}} \frac{d}{d r}\left(r^{N-1+(N-2) \alpha} f^{\prime}(r)\right) .
$$

The proof will be by induction. In the cases $k=], 2$ it was given in Sario-Wang [1]. For $k \geq 3$ we have the induction hypothesis

$$
\begin{aligned}
-\frac{1}{r^{N-1+N a}} \frac{d}{d r}\left(r^{N-1+(N-2) \alpha} f^{\prime}(r)\right) \\
\quad=\sum_{n=0}^{k-2}\left(a_{n} r^{(2 n-N+2)(\alpha+1)}+b_{n} r^{2 n(\alpha+1)}\right) .
\end{aligned}
$$

Here and later $a_{n}, b_{n}, C, c_{n}$, etc. are constants, not always the same. 
We obtain successively

$$
\begin{aligned}
& \frac{d}{d r}\left(r^{N-1+(N-2) \alpha} f^{\prime}(r)\right)=\sum_{n=0}^{k-2}\left(a_{n} r^{(2 n+2)(\alpha+1)-1}+b_{n} r^{(2 n+N)(\alpha+1)-1}\right) \\
& r^{N-1+(N-2) \alpha} f^{\prime}(r)=\sum_{n=0}^{k-2}\left(a_{n} r^{(2 n+2)(\alpha+1)}+b_{n} r^{(2 n+N)(\alpha+1)}\right)+C \\
& f^{\prime}(r)=\sum_{n=0}^{k-2}\left(a_{n} r^{(2 n+2-N)(\alpha+1)+2 \alpha+1}+b_{n} r^{2 n(\alpha+1)+2 \alpha+1}\right)+C r^{-N-(N-2) \alpha+1} \\
& f(r)=\sum_{n=0}^{k-1}\left(a_{n} r^{(2 n+2-N)(\alpha+1)}+b_{n} r^{2 n(\alpha+1)}\right)
\end{aligned}
$$

3. Let $S_{n m}=S_{n m}(\theta) ; n=1,2, \ldots ; m=1, \ldots, m_{n}$, be the (Euclidean) surface spherical harmonics. We do not include $n=0$ in our notation $S_{n m}$, as we treat constants as radial functions. For harmonic functions we know (loc. cit.) that $f(r) S_{n m} \in H\left(E_{\alpha}^{N}\right)$ for any $N$ and any $\alpha$ if and only if $f(r)=a r^{p_{n}}+b r^{q_{n}}$ where $a, b$ are arbitrary constants and

$$
\left\{\begin{array}{l}
p_{n}=\frac{1}{2}\left[-(N-2)(\alpha+1)+\sqrt{(N-2)^{2}(\alpha+1)^{2}+4 n(n+N-2)}\right] \\
q_{n}=\frac{1}{2}\left[-(N-2)(\alpha+1)-\sqrt{(N-2)^{2}(\alpha+1)^{2}+4 n(n+N-2)}\right]
\end{array}\right.
$$

4. For any $N, a, n>0,0 \leq j \leq k-2$,

$$
P_{n j}=\left(\frac{1}{2} N+j\right)(\alpha+1)+p_{n}, \quad Q_{n j}=\left(\frac{1}{2} N+j\right)(\alpha+1)+q_{n} .
$$

Define $n_{j}^{\prime}, n_{j}^{\prime \prime}$ by $P_{n_{j} j}^{\prime}=0, Q_{n_{j} j}^{\prime \prime}=0$. Then

$$
P_{n j} \neq 0 \text { and } Q_{n j} \neq 0 \text { for } N \geq 2 k \text {, any } a, n \text {. }
$$

For the proof, we observe that $P_{n j}=0$ implies

$$
\left[4(j+1)^{2}-(N-2)^{2}\right](\alpha+1)^{2}=4 n(n+N-2) .
$$

If $N \geq 2 k$,

$$
4(j+1)^{2} \leq 4 k^{2}-8 k+4 \leq(N-2)^{2} .
$$

Since our $n>0$, there are no roots. The proof of (5) for $Q_{n j}$ is identical. 
5. The equation $\Delta u=r^{p_{n_{j}^{\prime}}^{\prime}+(2 \alpha+2) j} S_{n_{j}^{\prime} m}^{\prime}$ has a solution

$$
u_{n_{j}^{\prime} m}^{\prime}=a r_{n_{j}^{\prime}}+(2 \alpha+2)(j+1) \log r \cdot S_{n_{j}^{\prime} m}^{\prime}
$$

and the equation $\Delta v=r^{q_{n j}^{\prime \prime}+(2 \alpha+2) j} S_{n_{j}^{\prime \prime} m}$ a solution

$$
v_{n_{j}^{\prime \prime} m}=b r_{n_{j}^{\prime \prime}}+(2 \alpha+2)(j+1) \log r \cdot S_{n_{j}^{\prime \prime} m}
$$

with $a, b$ certain constants. We see this by direct computation which is made easier by noting that $r_{n_{j}^{\prime}}^{p^{\prime}} S_{n_{j} m}^{\prime}$ and $r_{n_{j}}^{q_{\prime \prime}} S_{n_{j}^{\prime \prime} m}$ are harmonic. In this computation one observes that multiplying $u$ or $v$ by $r^{2 \alpha+2}$ raises its degree of polyharmonicity by one, and $\Delta\left(r^{2 \alpha+2} u\right)=$ const. $u$ +harmonic function.

6. It is easy to verify that for any $N, a \neq-1$, the equations

$$
\Delta u=r^{p_{n}+(2 \alpha+2) j} S_{n m}, \quad \Delta v=r^{q_{n}+(2 \alpha+2) j} S_{n m}
$$

have solutions $u_{n m}$ for $n \neq n_{j}^{\prime}$ and $v_{n m}$ for $n \neq n_{j}^{\prime \prime}$ given by

$$
u_{n m}=a r^{p_{n}+(2 \alpha+2)(j+1)} S_{n m}, \quad v_{n m}=b r^{q_{n}+(2 \alpha+2)(j+1)} S_{n m} .
$$

7. In the case $a=-1, j \geq 1$ we shall prove that

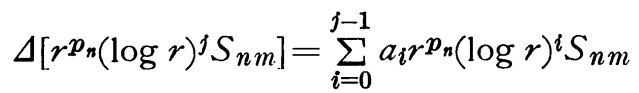

for certain constants $a_{i}$. In view of $\Delta \log r=\Delta r p_{n} S_{n m}=0$,

$$
\Delta\left(r^{p_{n}} \log r \cdot S_{n m}\right)=-2\left(\operatorname{grad} r^{p_{n}} \cdot \operatorname{grad} \log r\right) S_{n m}=-2 p_{n} r^{p_{n}} S_{n m} .
$$

A straightforward induction argument completes the proof.

8. For harmonic functions we know (loc. cit.) that given any $N$, $\alpha$, every $h \in H\left(E_{\alpha}^{N}\right)$ has an expansion

$$
h=\sum_{n=1}^{\infty} \sum_{m=1}^{m_{n}}\left(a_{n m} r^{p_{n}}+b_{n m} r^{q_{n}}\right) S_{n m}+\gamma_{1}(r) \text {. }
$$

We can now proceed to polyharmonic functions. For any $N$, any $u \in H^{k}\left(E_{\alpha}^{N}\right)$ has an expansion for $a \neq-1$ 


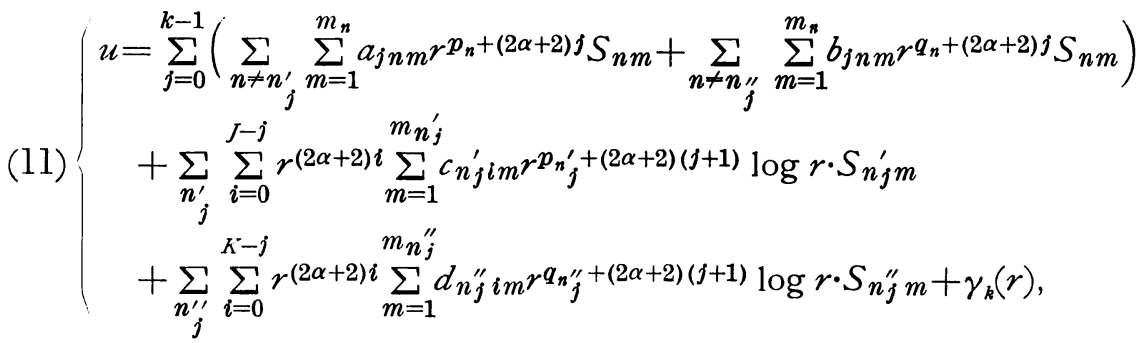

where $J=\max \left\{j \mid P_{n_{j j}}^{\prime}=0\right\}, K=\max \left\{j \mid Q_{n_{j}^{\prime \prime}}=0\right\}$. If $\alpha=-1$, then

$$
u=\sum_{j=0}^{k-1} \sum_{n=1}^{\infty} \sum_{m=1}^{m_{n}}\left(a_{j n m} r^{p_{n}}+b_{j n m} r^{q_{n}}\right)(\log r)^{j} S_{n m}+\gamma_{k}(r) .
$$

For the proof let $h=\Delta^{k-1} u$ have expansion (10). The proper coefficients of $u$ are obtained from (1)-(3), (6)-(9). The expansion of $h$ converges for every $r \in(0, \infty)$ and all $\theta$. Therefore,

$$
\begin{aligned}
& \lim _{n \rightarrow \infty}\left|\sum_{m=1}^{m_{n}} a_{n m} S_{n m}\right| \frac{1}{\boldsymbol{p}_{n}}=\lim _{n \rightarrow \infty}\left|\sum_{m=1}^{m_{n}} b_{n m} S_{n m}\right|-\frac{1}{\boldsymbol{q}_{n}}=0, \\
& \lim _{n \rightarrow \infty}\left|\sum_{m=1}^{m_{n}} a_{j n m} S_{n m}\right| \frac{1}{\boldsymbol{p}_{n}}=\lim _{n \rightarrow \infty}\left|\sum_{m=1}^{m_{n}} b_{j n m} S_{n m}\right|-\frac{1}{\boldsymbol{q}_{n}}=0,
\end{aligned}
$$

and the expansion of $u$ converges for all $(r, \theta)$. We apply the operator $\Delta^{k-1}$ term-by-term and obtain (10).

9. We continue with the proof of Theorem 1 and discuss first the case $a \neq-1$. If $j \neq n$ or $k \neq m$, then $S_{j k}$ and $S_{n m}$ are orthogonal with respect to the inner product $(\cdot, \cdot)$ :

$$
\left(S_{j k}, S_{n m}\right)=\int_{\partial B(0,1)} S_{j k} S_{n m} d \omega
$$

where $B(0,1)$ is the unit ball about the origin, and $d \omega$ is the Euclidean surface element of $\partial B(0,1)$.

If $u \in H^{k} B$, then $\left(u, S_{n m}\right)$ is bounded for any $(n, m)$. For $\alpha \neq-1$, $N \geq 2 k$,

$$
\left(u, S_{n m}\right)=\mathrm{const} \sum_{j=0}^{k-1}\left(a_{j n m} r^{p_{n}+(2 \alpha+2) \jmath}+b_{j n m} r^{q_{n}+(2 \alpha+2) \jmath}\right) .
$$


Because the right-hand side must be bounded for any choice of $r \in(0, \infty)$, either $a_{j n m}$ or $p_{n}+(2 \alpha+2) j$ vanishes, and either $b_{j n m}$ or $q_{n}+(2 a+2) j$ vanishes, for all $j$. We note that

$$
p_{n}+(2 \alpha+2) j=0 \quad \text { and } \quad q_{n}+(2 \alpha+2) j=0
$$

is equivalent with

$$
\left[(4 j+2-N)^{2}-(N-2)^{2}\right](a+1)^{2}=4 n(n+N-2) .
$$

If $N \geq 2 k, \quad\left[(4 j+2-N)^{2}-(N-2)^{2}\right] \leq 0$, and (13) has no solutions by virtue of $n>0$. Therefore, the coefficients $a_{j n m}, b_{j n m}$ vanish for all $(j, n, m)$.

We conclude that all terms in (1) and (2), except for the constant, vanish because, for fixed $N, \alpha$, they are unbounded. The proof of Theorem 1 is completed by using a similar argument for $a=-1$. In the case $a=-1$ the theorem is true for all $N$.

10. We proceed to show that $H^{k} B$-functions exist on the lower dimensional spaces for certain $a$. Examining the proof of Theorem 1 , we see that it would hold for $N<2 k$ if again (13) had no solutions; in fact, the terms involving $n_{j}^{\prime}$ and $n_{j}^{\prime \prime}$ would be eliminated as they are not bounded. Hence, we need only find out when (13) has solutions.

Theorem 2. For fixed $N, a \neq-1, N<2 k$, the generators of $H^{k} B$ are the $S_{n m}$ such that (13) holds.

Proof. That the $S_{n m}$ are $H^{k} B$-functions follows from (8). By solving the equations in (13) in the form

$$
\left(2 j+1-\frac{1}{2} N\right)(a+1)=-\frac{1}{2} \sqrt{(N-2)^{2}(a+1)^{2}+4 n(n+N-2)},
$$

we find that the solutions for $j=k-1, n \neq 0$, are

$$
\alpha=-1 \pm \sqrt{\frac{n(n+N-2)}{4 k^{2}-(4+2 N) k+2 N}} .
$$


11. One might suspect that the existence of $H^{k} B$-functions always implies that of nondegenerate $H^{h} B$-functions for $h>k$. However, we shall show:

Theorem 3. $E_{\alpha}^{N} \notin O_{H^{k} B}^{N_{k}}$ implies $E_{\alpha}^{N} \notin O_{H^{h} B}^{N_{B}}$ for all $h>k$ and all $N$ if $a=0$. There exist $E_{\alpha}^{N}$ for which this is no longer true.

Proof. If $a=0$, equation (14) reduces to $n=2 j+2-N$. Therefore, if there exists an $n$ satisfying this for $j=k-1$, there also exists an $n$ for all $h \geq k$.

To show that the above is not true for all $\alpha$, we choose $N=4$, $n=1$. For $j=3$ we then have $a=-1+8^{-\frac{1}{2}}$ whereas $j=4$ should give $6=n(n+2)$. Since no integer $n$ satisfies this equation, we conclude for the above $\alpha$ that $E_{\alpha}^{N} \in O_{H^{5} B} \backslash O_{H^{4} B}$.

\section{University of CAlifornia, Los Angeles}

\section{References}

1. L. Sario-C. Wang, Riemannian manifolds of dimension $N \geq 4$ without bounded biharmonic functions, J. London Math. Soc. (to appear).

2. - Generators of the space of bounded biharmonic functions, Math. Z. 127 (1972), 273-280. 